\title{
I-, 3-, and 5-year survival among early-stage lung cancer patients treated with lobectomy vs SBRT
}

This article was published in the following Dove Press journal: Lung Cancer:Targets and Therapy

\author{
Denise Albano' \\ Thomas Bilfinger' \\ Barbara Nemesure ${ }^{2}$ \\ 'Department of Surgery, Health \\ Sciences Center, Stony Brook \\ Medicine, Stony Brook, NY, USA; \\ ${ }^{2}$ Department of Family, Population and \\ Preventive Medicine, Health Sciences \\ Center, Stony Brook Medicine, Stony \\ Brook, NY, USA
}

Background: Lobectomy has traditionally been recommended for fit patients diagnosed with early-stage non-small-cell lung cancer (NSCLC). Recently, however, stereotactic body radiotherapy (SBRT) has been introduced as an alternative treatment option. The purpose of this investigation is to compare survival outcomes for individuals with stage I/II NSCLC treated with lobectomy vs SBRT.

Methods: This retrospective study included 191 patients (100 surgery, 91 SBRT) identified through the Lung Cancer Evaluation Center, Stony Brook, NY, between 2008 and 2012. Survival and recurrence rates were compared using Kaplan-Meier curves, log-rank tests, and Cox proportional hazard models to adjust for possible confounders. A subset of cases was propensitymatched to address potential differences in health status between groups.

Results: 1-, 3-, and 5-year survival outcomes were significantly better among patients undergoing lobectomy vs SBRT. Survival rates at 3 years were $92.8 \%$ and $59.0 \%(p<0.001)$ in the 2 groups, respectively. Propensity-matched analyses indicated similar findings. Recurrence rates were likewise lower among patients undergoing surgery $(7.1 \%$ vs $21.0 \%, p<0.01$ at 3 years); however, statistical significance was not maintained in the propensity-matched analysis.

Conclusion: These findings add to a growing evidence base supporting the use of lobectomy vs SBRT in the treatment of lung cancer among healthy, early-stage NSCLC patients.

Keywords: SBRT, lobectomy, survival

\section{Introduction}

Lung cancer is the leading cause of cancer mortality worldwide, accounting for 1.3 million deaths each year. ${ }^{1}$ In 2018, an estimated 234,030 new cases of lung cancer will be diagnosed in the United States and 154,050 disease-related deaths are expected to occur. ${ }^{2}$ The recommended treatment for early-stage non-small-cell lung cancer (NSCLC) has historically been lobectomy with mediastinal lymph node dissection, ${ }^{3}$ and according to current National Comprehensive Cancer Network (NCCN) guidelines, surgery remains the best therapeutic option to date. ${ }^{4,5}$

Stereotactic body radiation therapy (SBRT) has traditionally been used as a consolidated treatment therapy for patients with medically inoperable lung cancer ${ }^{6}$ and in patients considered to be at high-risk as a result of comorbidities, poor pulmonary function, and/or advanced age. ${ }^{7}$ Recently, SBRT is being offered as a treatment option for healthy patients with early-stage lung cancer. ${ }^{8,9}$ It remains unclear, however, how survival and recurrence outcomes among patients being treated by SBRT compare to such rates among similar patients treated by surgical resection. The purpose of this
Correspondence: Barbara Nemesure Department of Family, Population and Preventive Medicine, Stony Brook Medicine, Nicolls Rd HSC, L3, Stony Brook, NY I I794-8036, USA

$\mathrm{Tel}+\mid$ 63| 444 |293

Email Barbara.Nemesure@ stonybrookmedicine.edu 
investigation was to evaluate 1-, 3-, and 5-year survival and recurrence outcomes between healthy patients with biopsyproven clinical stage I/II NSCLC undergoing primary treatment with surgical resection compared to SBRT.

\section{Methods}

\section{Data source/patient selection}

A retrospective study of 191 biopsy-proven, early-stage NSCLC patients treated at Stony Brook Cancer Center's Lung Cancer Evaluation Center between 2008 and 2012 was conducted to assess survival and recurrence outcomes between two different treatment modalities for patients with stage I/II lung cancer. This investigation included 100 patients treated by surgical lobectomy and 91 patients treated nonsurgically with SBRT. All patients were clinically staged by physical examination, imaging and biopsy results. Eligible patients underwent positron emission tomography-computed tomography (PETCT), which did not show any evidence of metastatic adenopathy. Further, surgical patients also received pathological staging with lymph node status. For this investigation, staging was based on pathological report for the surgical cases and based on PET and biopsy including endobronchial ultasound for those treated by SBRT. Exclusion criteria included patients with small-cell lung cancer or other cancers that had metastasized to the lung, patients staged with IIB cancer or greater, patients who underwent resection for benign disease, and those without available preoperative staging chest PET/CT scans.

All patients were evaluated by surgeons prior to deciding their course of treatment. The multidisciplinary workup included pulmonary function testing and complete cardiac status, as well as performance status determinations. Every patient was provided information on surgical interventions, as well as SBRT, and subsequently decided (with the assistance of the clinical care team) on their individualized treatment plan.

Data abstracted for each patient included date of diagnosis, age at diagnosis, gender, histology (squamous cell, adenocarcinoma, other type), smoking history (current, former, never), history of comorbidities including COPD/asthma, history of hypertension and diabetes, location of the lesion (left lower lobe, left middle lobe, left upper lobe, right lower lobe, right middle lobe, right upper lobe), survival status, duration of survival, recurrence status, and time to recurrence.

\section{Treatment options}

\section{Surgical technique}

Lobectomy was performed with frozen section analysis for all bronchopulmonary, hilar, and mediastinal lymph nodes.
Once the tumor was resected, pathological confirmation of T1 and NSCLC status was obtained, and histological information from the lymph nodes was used for accurate staging. Patients were hospitalized for between 3 and 5 days, on average. Follow-up care included a CT scan after 1 month and semi-annually for 2 additional years, followed by CT scans every year for 5 years in accordance with NCCN guidelines. A PET scan was also performed at 12 months and again at 24 months for surveillance.

\section{Radiation technique}

The lung cancer SBRT treatment regimen at the Stony Brook Cancer Center follows recommendations provided by the American College of Radiology and the American Society of Radiation Oncology. Patients receive 12 Gy for 4 treatments delivering high-dose radiation using multiple conformal coplanar and non-coplanar beams. This technique has been reported to concentrate the prescribed radiation dose to the tumor more precisely than conventional radiation therapy. ${ }^{7}$ Patients undergoing SBRT have 2 treatments the first week and 2 treatments the following week. Patients are subsequently followed with CT at 3, 6, 12, 18, and 24 months and then yearly as per NCCN guidelines. A PET is performed at 6,12 , and 24 months for surveillance.

Data used in this investigation did not include any personal identifiers, thereby ensuring patient confidentiality. As such, patient consent was not required by the Stony Brook University Institutional Review Board and Committee on Research Involving Human Subjects, which approved this investigation (approval number 2016-3701-F).

\section{Data analyses}

This investigation included 191 cases $(n=100$ surgery, $n=91$ SBRT) with known survival status at 1-year post-treatment. $\chi^{2}$ tests and independent sample $t$-tests were used to evaluate differences between groups for categorical and continuous data, respectively. Kaplan-Meier (K-M) curves and logrank tests were presented to represent differences in 1-, 3-, and 5-year survival and recurrence outcomes between treatment groups. Patients for whom no event occurred within the specified time frame were right-censored. $\mathrm{K}-\mathrm{M}$ analyses were conducted independently at 1, 3, and 5 years based on available data for the time periods of interest. Cox proportional hazard $(\mathrm{CPH})$ models were used to assess any possible effects of treatment on survival. The $\mathrm{CPH}$ models were adjusted for age, gender, smoking status, histology, and any comorbidity. HR and 95\% CI are presented. Additionally, a propensity-matched analysis was performed to 
evaluate 1-, 3-, and 5-year survival and recurrence outcomes between treatments groups among cases matched on factors found to be significantly different between groups. These factors included age at diagnosis, gender, histology, and any comorbidity. Propensity scores were calculated for each patient using logistic regression with the treatment group defined as the dependent variable and all noted covariates included as independent factors. Matching was based on the "nearest neighbor" approach with a caliper distance of 0.15 . To maximize sample size, "one to many" matching was applied in which a single SBRT patient was potentially matched to $>1$ surgical patient with similar propensity scores. The standardized mean difference between groups was $\leq 0.1$ for each covariate, indicating a well-matched sample. The final propensity-based model yielded a total of $\mathrm{n}=76$ surgery patients and $\mathrm{n}=56$ SBRT patients who were propensity matched and served as the basis for the survival and recurrence analyses at year 1 . Subsets of these patients with available data were evaluated at 3 and 5 years, as well.

These analyses were carried out using SPSS version 21 (IBM Corporation, Armonk, NY, USA).

\section{Results}

This investigation included 191 patients with early-stage NSCLC treated by either surgical resection or SBRT. Table 1 presents demographic characteristics of patients stratified by group (lobectomy vs SBRT). Patients undergoing surgery were younger, on average, than those receiving SBRT (65.5 vs 73.7 years, $p<0.01)$ and were more likely to be female $(p=0.03$ ). Additionally, approximately three-quarters of the SBRT group had a noted comorbidity, which was significantly higher than the $64 \%$ of any such comorbidity in the surgery group ( $p=0.05$ ). Although smoking status at the time of diagnosis and the location of the lesion were not significantly different between groups, the distribution of histology type varied between the surgery vs SBRT patients. Adenocarcinoma and squamous cell cancers collectively represented $98 \%$ of the histologic findings among those undergoing resection compared with $89 \%$ among those receiving radiation therapy ( $p=0.03$ ).

Kaplan-Meier curves for all study participants are displayed in Figure 1. A significant survival benefit is noted for patients undergoing lobectomy compared to those receiving SBRT $(p<0.001)$.

$\mathrm{CPH}$ models were used to estimate the effect of treatment on survival outcomes, while adjusting for age at diagnosis, gender, smoking status, histology, and any comorbidity. The finding indicate that surgical resection significantly improved survival compared to SBRT (HR $=0.19,95 \%$ CI $[0.09,0.38])$. Furthermore, none of the additional factors

Table I Demographic characteristics of lobectomy vs SBRT among early-stage lung cancer patients

\begin{tabular}{|c|c|c|c|}
\hline Characteristic & Lobectomy $(\mathrm{N}=100)$ & SBRT $(\mathbf{N}=91)$ & $P$-value \\
\hline Age at diagnosis, years & $65.5 \pm 9.9$ & $73.7 \pm 9.8$ & $<0.01$ \\
\hline Gender, \% male & 35.0 & 50.5 & 0.03 \\
\hline \multicolumn{4}{|l|}{ Histology } \\
\hline Adenocarcinoma & 77.0 & 63.7 & 0.03 \\
\hline Squamous cell & 21.0 & 25.3 & \\
\hline Other & 2.0 & 11.0 & \\
\hline \multicolumn{4}{|l|}{ Smoking status } \\
\hline Current & 45.0 & 38.5 & 0.06 \\
\hline Former & 53.0 & 51.6 & \\
\hline Never & 2.0 & 9.9 & \\
\hline COPD, \% & 46.0 & 59.0 & 0.08 \\
\hline Hypertension, \% & 34.0 & 21.7 & 0.07 \\
\hline Diabetes, \% & 10.0 & 7.2 & 0.51 \\
\hline Any comorbidity, \% & 64.0 & 77.1 & 0.05 \\
\hline \multicolumn{4}{|l|}{ Location } \\
\hline LLL & 26.0 & 20.9 & 0.24 \\
\hline LML & - & - & \\
\hline LUL & 25.0 & 24.2 & \\
\hline RLL & 22.0 & 18.7 & \\
\hline RML & 5.0 & 1.1 & \\
\hline RUL & 22.0 & 35.1 & \\
\hline
\end{tabular}

Abbreviations: LLL, left lower lobe; LML, left middle lobe; LUL, left upper lobe; RLL, right lower lobe; RML, right middle lobe; RUL, right upper lobe; SBRT, stereotactic body radiation. 


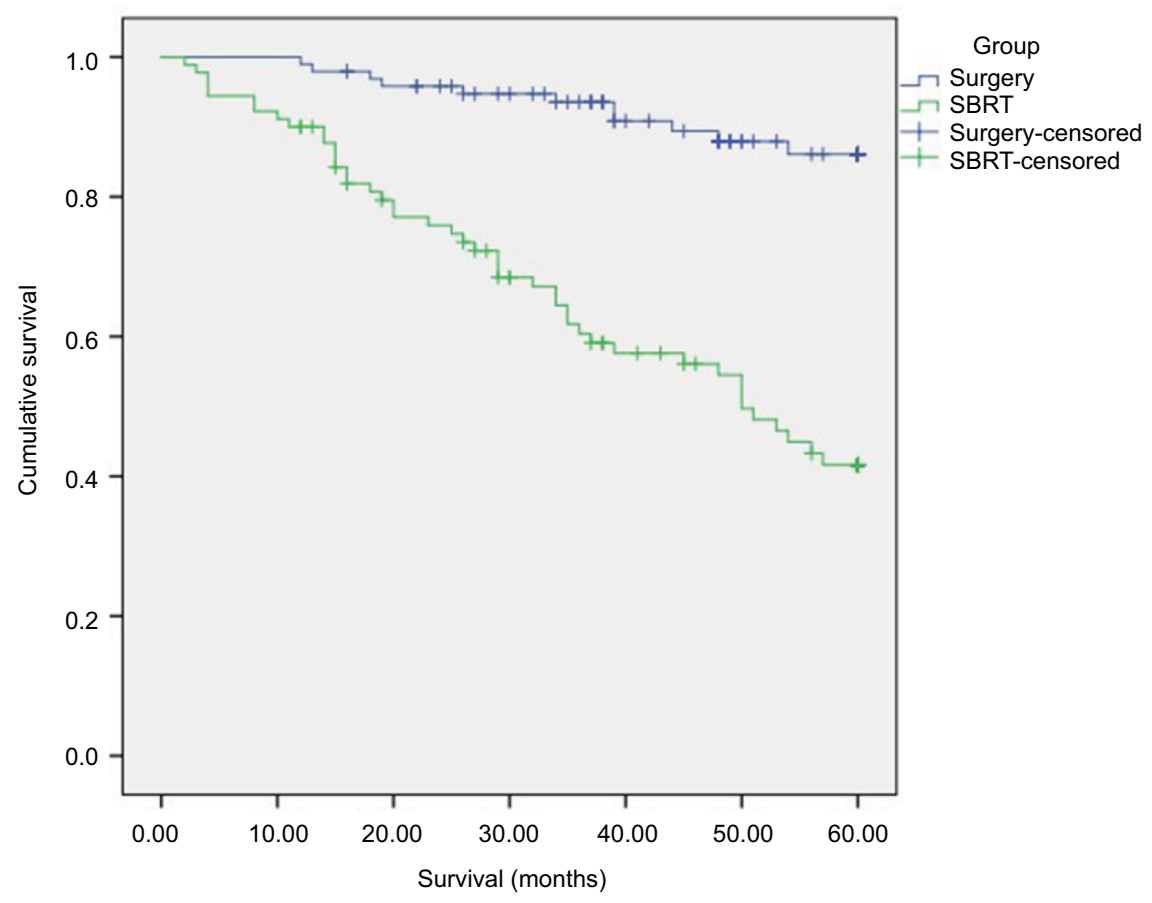

Figure I 5-Year K-M survival curves for patients treated by lobectomy vs SBRT. Abbreviations: K-M, Kaplan-Meier; SBRT, stereotactic body radiation.

included in the model were found to be significant predictors for survival.

Table 2 presents the 1-, 3-, and 5-year survival outcomes for each treatment group. Both 1- and 5-year survival outcomes were significantly improved in patients in the resection group compared to those treated by SBRT. Among all cases, survival rates 1-year post surgery were $99.0 \%$ for those resected compared to $90.1 \%$ in the radiation group $(p=0.01)$. At 3 years after treatment, survival rates among the patients undergoing surgery remained relatively constant $(99.0 \%$ to $92.8 \%$ ), while corresponding rates in the SBRT group decreased by approximately $35 \%$ (90.1\% to $59.0 \%)$. Survival was significantly better among surgery patients, with 5-year survival found to be $>2$ times higher among patients who underwent lobectomy compared to those receiving SBRT $(80.4 \%$ vs $36.8 \%, p<0.001)$. When cases were propensity matched for age at diagnosis, gender, histology, and any comorbidity, the findings were similar. At 5 years, the survival rate among patients undergoing surgery was $76.2 \%$ vs $30.0 \%$ among those receiving SBRT $(p<0.001)$ (Table 2$)$.

Table 3 presents the 1-, 3-, and 5-year recurrence for patients treated by lobectomy vs SBRT. At year 1, lung cancer recurrence rates were 4 times higher among patients receiving SBRT compared to those undergoing surgical resection $(8.8 \%$ vs $2.0 \%, p=0.04)$. At 5 years, more than one-quarter of patients treated with radiation were found to have had a recurrence compared to $12.3 \%$ among those treated by surgery $(p=0.05)$. When the cases in both groups were propensity matched, the findings showed similar rates of recurrence at 1,3 , and 5 years, but the differences between treatment modalities did not achieve statistical significance, likely due to the reduced sample sizes.

\section{Discussion}

Data are limited regarding the efficacy of surgical resection vs SBRT in the treatment of fit patients diagnosed with early-stage lung cancer. Although some studies have reported improved outcomes among patients treated with lobectomy compared to SBRT, ${ }^{7,8,10}$ others have found no significant differences in survival and/or recurrence between the 2 treatment modalities. ${ }^{11}$ Investigations to date have been challenged by issues related to sample selection biases, patient recruitment, and other factors. The present study of 191 patients treated for stage I/II lung cancer at the Stony Brook Cancer Center found a significantly higher survival rate and fewer recurrences, both short term and long term, among patients undergoing surgical resection compared to those treated by SBRT. Although lobectomy appears to yield superior outcomes to SBRT, prospective studies are necessary to more accurately quantify the magnitude of the differences 
Table 2 I-, 3-, and 5-year survival rates among patients with early-stage lung cancer stratified by treatment modality

\begin{tabular}{|c|c|c|c|}
\hline Survival time & Lobectomy (\% survived) & SBRT (\% survived) & $P$-value \\
\hline \multicolumn{4}{|l|}{ All cases ${ }^{a}$} \\
\hline I-year & 99.0 & 90.1 & 0.01 \\
\hline 3-year & 92.8 & 59.0 & $<0.001$ \\
\hline 5 -year & 80.4 & 36.8 & $<0.001$ \\
\hline \multicolumn{4}{|c|}{ Propensity-matched cases ${ }^{\mathrm{b}}$} \\
\hline I-year & 100.0 & 94.6 & 0.04 \\
\hline 3-year & 92.2 & 56.5 & $<0.001$ \\
\hline 5-year & 76.2 & 30.0 & $<0.001$ \\
\hline
\end{tabular}

Notes: all cases - year I: $n=100$ surgery, $n=91$ SBRT; year $3: n=83$ surgery, $n=78$ SBRT; year $5: n=56$ surgery, $n=68$ SBRT. bPropensity-matched cases were matched on age at diagnosis, gender, histology, and any comorbidity. Year I: $n=76$ surgery, $n=56$ SBRT; year 3: $n=64$ surgery, $n=46$ SBRT; year 5: $n=42$ surgery, $n=40$ SBRT.

Abbreviation: SBRT, stereotactic body radiation.

Table 3 I-, 3-, and 5-year recurrence rates among patients with early-stage lung cancer stratified by treatment modality

\begin{tabular}{|c|c|c|c|}
\hline Recurrence time & Lobectomy n (\%) recurrences & SBRT n (\%) recurrences & $P$-value \\
\hline \multicolumn{4}{|l|}{ All cases ${ }^{a}$} \\
\hline I-year & $2(2.0)$ & $8(8.8)$ & 0.04 \\
\hline 3-year & $6(7.1)$ & $17(2 \mid .0)$ & 0.01 \\
\hline 5 -year & $7(12.3)$ & $20(26.0)$ & 0.05 \\
\hline \multicolumn{4}{|c|}{ Propensity-matched cases ${ }^{\mathrm{b}}$} \\
\hline I-year & $2(2.6)$ & $4(7.1)$ & 0.22 \\
\hline 3-year & $5(7.8)$ & $9(18.8)$ & 0.08 \\
\hline 5-year & $6(14.3)$ & $10(22.2)$ & 0.34 \\
\hline
\end{tabular}

Notes: ${ }^{\text {AAll }}$ cases - year I: $n=100$ surgery, $n=9$ I SBRT; year $3: n=84$ surgery, $n=8$ I SBRT; year $5: n=57$ surgery, $n=77$ SBRT. bPropensity-matched cases were matched on age at diagnosis, gender, histology, and any comorbidity. Year I: $n=76$ surgery, $n=56$ SBRT; year 3: $n=64$ surgery, $n=48$ SBRT; year 5: $n=42$ surgery, $n=45$ SBRT.

Abbreviation: SBRT, stereotactic body radiation.

in both survival and recurrence among early-stage lung cancer patients treated by these modalities.

\section{Survival outcomes}

Several studies have compared survival rates between early-stage lung cancer patients undergoing surgical resection with those receiving SBRT, and the findings have been somewhat inconsistent. In one study of 58 operable, stage I NSCLC patients (31 SABR, 27 surgery), the 3-year survival outcomes were found to be superior in the SABR group compared to those undergoing lobectomy ( $95 \%$ vs $79 \%$, respectively). ${ }^{9}$ Surgical resection, however, resulted in significantly improved outcomes in several other investigations. In a study including 183 elderly ( $\geq 75$ years) patients, a survival advantage was reported among 154 cases treated with lobectomy compared to 35 treated with SBRT, with overall 5-year survival rates of $67.6 \%$ and $43.8 \%$, respectively. ${ }^{11}$

Likewise, in another investigation, data were combined from 4 institutions and included 132 stage I lung cancer patients who underwent lobectomy and 137 who received SBRT. ${ }^{10}$ Patients in both groups were matched on histology, Charleston comorbidity index, tumor size, history of diabetes, and/or hypertension and forced expiratory volume in 1 second. In the matched-pair analysis, the overall 5-year survival was superior in the surgical than the SBRT group (69.2\% vs $33.7 \%$, respectively; $p=0.004)$. However, it was noted that after adjustment for treatment selection, the survival outcomes were found to be similar in both groups.

In a third study, survival outcomes among 462 patients with stage I lung cancer who underwent surgery were compared to corresponding outcomes among 76 lung cancer cases who received SBRT. ${ }^{7}$ Surgery patients were reported to be younger and more fit than those in the radiation group, with fewer comorbidities and better pulmonary function test scores. In an unmatched analysis, the 3-year overall survival was significantly better in the surgery than the SBRT group (68\% and $32 \%$, respectively). However, after propensity matching 57 high-risk surgical patients to 57 SBRT patients, overall survival at 3 years was not found to be statistically significant ( $54 \%$ vs $38 \%$ ).

One of the largest studies to date comparing long-term survival outcomes of healthy lung cancer patients with stage I disease resulted from a retrospective investigation using data from the National Cancer Database. ${ }^{8}$ A total of 13,562 
clinical stage I patients treated with lobectomy were found to have significantly better outcomes than 1,781 patients receiving SBRT $(p<0.001)$. Additionally, in a propensitymatched analysis of 1,781 pairs of patients within the same study, surgery remained superior to radiation treatment with 5 -year survival rates of $59 \%$ vs $29 \%$ ( $p<0.001)$, respectively.

Results from the present investigation are consistent with other reports supporting the survival benefit of surgery over SBRT among early-stage NSCLC patients; however, the magnitude of the survival rates found in this investigation were significantly higher than those presented in other reports. The unmatched 3-year survival for the lobectomy and SBRT groups in the current study were $93 \%$ and $59 \%$, respectively, compared to $68 \%$ and $32 \%$ in the study reported by Crabtree et al. ${ }^{7}$ Similar survival outcomes were also found with the propensity-matched analyses in both studies $(92 \%$ and $57 \%$ survival at 3 years in the present investigation; 54\% and $38 \%$ survival at 3 years in the study reported by Crabtree et $\mathrm{al}^{7}$ ). Additionally, our 5-year survival rates for the SBRT group in a propensity-matched analysis were consistent with those originating from the National Cancer Database $(30 \%$ vs 29\%); however, 5-year survival tended to be improved among patients in the present investigation compared to similarly matched lobectomy patients from the National Cancer Database (76\% vs 59\%). These findings add to the growing evidence base to support the use of lobectomy over SBRT in the treatment of early-stage lung cancer patients who are deemed to be fit for surgery, however, highlight the need for additional studies to better quantify the magnitude of the differences.

\section{Recurrence outcomes}

To date, only a limited number of reports describe recurrences among early-stage lung cancer patients treated by lobectomy vs SBRT, and the findings have been inconsistent. In one study by Varlotto et al, ${ }^{10}$ which included 132 cases treated with lobectomy and 137 with SBRT, both total recurrence and locoregional recurrence outcomes were found to be similar. Total recurrence control at 5 years was achieved in $55.5 \%$ of surgical patients and $83.3 \%$ of those receiving stereotactic radiation $(p=0.258)$. Similarly in a study of 58 stage I healthy NSCLC patients, $86 \%$ of SABR patients were recurrence-free at 3 years compared to $80 \%$ of the patients undergoing lobectomy. This difference was not statistically significant $(p=0.54)$.

In a second study by Crabtree et al, ${ }^{7}$ which included 462 surgery and 76 SBRT patients with stage IA disease, 3-year local tumor control was found to be significantly better among those undergoing surgery compared to those receiving SBRT ( $96 \%$ vs $89 \%, p=0.04)$. It was noted, however, that no such differences were observed for patients with stage IB cancer treated with either modality ( $p=0.89$ ). Further, a propensity match of 57 patients undergoing resection and 57 receiving SBRT also showed no difference of local recurrence control at 3 years $(88 \% \text { vs } 90 \%)^{7}$

Findings from the present study suggest better tumor control (fewer recurrences) among patients undergoing lobectomy compared to those receiving SBRT. At 3 years, recurrence control was $93 \%$ and $79 \%(p=0.01)$ in each group, respectively, which is consistent with rates reported by Crabtree et $\mathrm{al}^{7}$ ( $96 \%$ and $89 \%$, respectively). At 5 years, control was achieved for $88 \%$ and $74 \%(p=0.05)$ among those receiving the respective treatment modalities in the present investigation. After propensity matching of cases in both groups, 5-year recurrence rates tended to be higher among patients receiving SBRT compared to lobectomy; however, the findings did not achieve statistical significance, likely due to the reduced sample size in the latter analysis. To our knowledge, comparable 5-year recurrence data have not been reported by other studies to date. Additional prospective investigations are required to better quantify the true magnitude of the differences in outcomes between the 2 groups.

\section{Limitations}

This study has several limitations including its reduced sample size, retrospective study design, and unavailable data for factors that may influence outcomes. These include (but are not limited to) pulmonary function tests and lack of pathological staging information for patients receiving SBRT. Although it would be optimal to compare lobectomy vs SBRT outcomes in a controlled randomized trial, such attempts by the American College of Surgeons Oncology Group and others have not been successful to date, mainly due to challenges with accruing patients. ${ }^{8,12,13}$

Given the observational nature of studies designed to evaluate the efficacy of the 2 treatment modalities, it is often difficult to identify comparable patients in each group. There is a fair amount of heterogeneity among patients with regard to age, pulmonary function, and other factors. Typically, patients receiving SBRT are disproportionately less healthy than surgical candidates, thus introducing inherent biases into the investigation. ${ }^{6}$ Since comorbidities have been associated with worse health outcomes and more complex clinical management, ${ }^{14}$ we adjusted for such comorbidities 
(COPD/asthma, hypertension and diabetes), in our analyses. In addition, to further address possible sample selection biases, we also performed a propensity-matched analysis, which is designed to account for the imbalance between groups. ${ }^{15}$ We matched the surgical and SBRT patients on age at diagnosis, gender, histology, and any comorbidity, and the findings were similar to those based on the full cohort. At 5 years, the survival rate among patients undergoing surgery was $76.2 \%$ vs $30.0 \%$ among those receiving SBRT $(p<0.001)$.

Three randomized trials were not completed due to poor accrual - primarily because of bias of investigator. Our institution treatment selection is also biased - surgical patients have better pulmonary and general function, and SBRT patients have poor functional status. Because SBRT showed a strong survival benefit in inoperable patients, it is projected to be at least the same in operable patients.

\section{Conclusion}

Our study comparing survival and recurrence rates among early-stage lung cancer patients treated by lobectomy vs SBRT suggests that lobectomy leads to improved outcomes in fit patients undergoing surgical resection. Additional studies, including well-designed and sufficiently powered randomized trials, are necessary to better quantify the suggested survival benefit.

\section{Disclosure}

The authors report no conflicts of interest in this work.

\section{References}

1. Reck M, Popat S, Reinmuth N, et al; ESMO Guidelines Working Group. Metastatic non-small-cell lung cancer (NSCLC): ESMO Clinical Practice Guidelines for diagnosis, treatment and follow-up. Ann Oncol. 2014;25(Suppl 3):iii27-iii39.
2. ACS. ACS 2018 Facts and Figures; 2018. Available from: https://www. cancer.org/content/dam/cancer-org/research/cancer-facts-and-statistics/ annual-cancer-facts-and-figures/2018/cancer-facts-and-figures-2018. pdf. Accessed August 2, 2018.

3. Rusthoven CG, Kavanagh BD, Karam SD. Improved survival with stereotactic ablative radiotherapy (SABR) over lobectomy for early stage non-small cell lung cancer (NSCLC): addressing the fallout of disruptive randomized data. Ann Transl Med. 2015;3(11):149.

4. NCCN. NCCN Guidelines (2017); 2017. Available from: https://www. nccn.org/patients/guidelines/lung_screening/index.html\#16. Accessed August 30, 2016.

5. Vansteenkiste J, De Ruysscher D, Eberhardt WE, et al. Early and locally advanced non-small-cell lung cancer (NSCLC): ESMO Clinical Practice Guidelines for diagnosis, treatment and follow-up. Ann Oncol. 2013;24(Suppl 6):vi89-vi98.

6. Ricardi U, Badellino S, Filippi AR. Stereotactic radiotherapy for early stage non-small cell lung cancer. Radiat Oncol J. 2015;33(2):57-65.

7. Crabtree TD, Denlinger CE, Meyers BF, et al. Stereotactic body radiation therapy versus surgical resection for stage I non-small cell lung cancer. J Thorac Cardiovasc Surg. 2010;140(2):377-386.

8. Rosen JE, Salazar MC, Wang Z, et al. Lobectomy versus stereotactic body radiotherapy in healthy patients with stage I lung cancer. JThorac Cardiovasc Surg. 2016;152(1):44-54.e9.

9. Chang JY, Liu YH, Zhu Z, et al. Stereotactic ablative radiotherapy: a potentially curable approach to early-stage multiple primary lung cancer. Cancer. 2013;119(18):3402-3410.

10. Varlotto J, Fakiris A, Flickinger J, et al. Matched-pair and propensity score comparisons of outcomes of patients with clinical stage I nonsmall cell lung cancer treated with resection or stereotactic radiosurgery. Cancer. 2013;119(15):2683-2691.

11. Nakagawa T, Negoro Y, Matsuoka T, Okumura N, Dodo Y. Comparison of the outcomes of stereotactic body radiotherapy and surgery in elderly patients with cT1-2N0M0 non-small cell lung cancer. Respir Investig. 2014;52(4):221-226.

12. Chang JY, Senan S, Paul MA, et al. Stereotactic ablative radiotherapy versus lobectomy for operable stage I non-small-cell lung cancer: a pooled analysis of two randomised trials. Lancet Oncol. 2015;16(6):630-637.

13. Guckenberger M. Dose and fractionation in stereotactic body radiation therapy for stage I non-small cell lung cancer: lessons learned and where do we go next? Int J Radiat Oncol Biol Phys. 2015;93(4):765-768.

14. Valderas JM, Starfield B, Sibbald B, Salisbury C, Roland M. Defining comorbidity: implications for understanding health and health services. Ann Fam Med. 2009;7(4):357-363.

15. Sepesi B, Rice DC, Heymach JV, Vaporciyan AA, Swisher SG. Stage I lung cancer - to operate or to radiate? That is the question. J Thorac Dis. 2016;8(9):2324-2327.
Lung Cancer: Targets and Therapy

\section{Publish your work in this journal}

Lung Cancer: Targets and Therapy is an international, peer-reviewed, open access journal focusing on lung cancer research, identification of therapeutic targets and the optimal use of preventative and integrated treatment interventions to achieve improved outcomes, enhanced survival and quality of life for the cancer patient. Specific topics covered in the journal include: Epidemiology, detection and screening Cellular research and biomarkers; Identification of biotargets and agents with novel

Submit your manuscript here: https://www.dovepress.com/lung-cancer-targets--therapy-journa
Dovepress

mechanisms of action; Optimal clinical use of existing anticancer agents, including combination therapies; Radiation and surgery; Palliative care; Patient adherence, quality of life, satisfaction; Health economic evaluations. The manuscript management system is completely online and includes a very quick and fair peer-review system. Visit http://www.dovepress.com/testimonials.php to read real quotes from published authors. 